\title{
Metodología de enseñanza docente en el proceso educativo virtual, carrera de pedagogía de la historia y las ciencias sociales
}

\section{Methodology of teaching in the virtual educational process, career of pedagogy of history and social sciencies}

\author{
Rómulo Arteño Ramos ${ }^{1}$ \\ Mery Elizabeth Zabala Machado ${ }^{2}$ \\ Deysi Rosario Basantes Moscoso ${ }^{3}$ \\ Universidad Nacional de Chimborazo
}

\section{RESUMEN}

En el presente trabajo se plantea innovar la metodología de trabajo docente con el uso de la plataforma Moodle en el desarrollo de la actividad curricular de la Carrera de Pedagogía de la Historia y las Ciencias Sociales de la 1 Rómulo Arteño Ramos Profesor investigador, Magister en Geografía, Universidad Nacional de Chimborazo, Riobamba- Ecuador grupo de investigación Puruhá, correo rramos@unach.edu.ec ORCID: https://orcid. org/0000-0001-9606-6546

2 Mery Elizabeth Zabala Machado Profesora investigadora Magister en currículo carrera de Ciencias Sociales Universidad Nacional de Chimborazo, Riobamba -Ecuador correo mzabala@unach.edu.ec https://orcid.org/ 00000002-7559-278X

3 Deysi Rosario Basantes Moscoso, Profesora investigadora Magister en Docencia mención intervención Psicopedagógica Universidad Nacional de Chimborazo, Riobamba -Ecuador, correo: dbasantes@unach.edu.ec ORCID: https://orcid.org/0000-0001-8742-8226
Universidad Nacional de Chimborazo, partiendo de la calidad de liderazgo del aprendizaje para lograr la vinculación entre la actividad del docente y la del estudiante. Se pretende mirar a la historia y a la geografía, no como proceso de memorización de nombre y fechas, sino como conflicto socio cognitivo; es decir, como búsqueda del saber a través de un entorno virtual de aprendizaje que contengan recursos y actividades accesibles a los estudiantes.

PALABRAS CLAVE: Plataforma Moodle, metodología, proceso educativo. 


\section{SUMMARY}

In this work, it is proposed to innovate the teaching work methodology with the use of the Moodle platform in the development of the curricular activity of the History and Social Sciences Pedagogy Career of the National University of Chimborazo, based on the quality of learning leadership to achieve the link between the activity of the teacher and that of the student. It is intended to look at history, not as a process of memorizing names and dates, but as a socio-cognitive conflict; that is, as a search for knowledge through a virtual learning environment that contains resources and activities accessible to students.

KEYWORDS: Moodle platform, methodology, educational process.

\section{INTRODUCCIÓN}

Analizamos el Modelo Educativo de la Universidad Nacional de Chimborazo que plantea la necesidad de un trabajo docente acorde a las exigencias actuales, dentro de un ámbito disciplinar, pedagógico-didáctico y tecnológico que desarrolle competencias orientadas a una realidad histórica, social, económica, geopolítica, espacial y sobre todo educativa.

Analizado el marco teórico entonces, se plantea que la innovación educativa está orientada precisamente a la ejecución de metodológicas activas fundamentadas en aprendizajes basados en problemas, en aprendizajes por proyectos, en aprendizaje cooperativo y en un aprendizaje por descubrimiento inductivo, con métodos narrativos interrogativos, estudios de casos, y método científico.

El campo de la Filosofía, Historia y las Ciencias Sociales es un tema bastante complejo, pues no existe consenso en las llamadas ciencias del espíritu, ciencias culturales, humanas y sociales, lo que en la actualidad ha sido tema de los debates epistemológicos más importantes en educación y sobre todo al analizar la metodología que los docentes deben emplear considerando los modos de producción científica de las ciencias experimentales y de las ciencias sociales o ciencias humanas. Ésta en los últimos tiempos han seguido el paradigma de la ciencia clásica, se consideraba al sujeto cognoscente como un simple observador, separado del objeto, despojado de sus emociones e intereses y creencias, es por eso que se considera necesario y de acuerdo a las políticas nacionales y los diversos lineamientos de los organismo de control de la educación Superior, la necesidad de reinsertar al sujeto educativo en el paradigma de la complejidad sistémica, con una reorganización del currículo donde se priorice la metodología de trabajo docente.

La Universidad debe convertirse en gestora del conocimiento y transformadora del quehacer educativo, con la finalidad de responder a las actuales exigencias y expectativas de la comunidad y sociedad, para articular las distintas prioridades establecidas en la Constitución de la República de Ecuador: Políticas y Lineamientos Nacionales, Plan Nacional de Desarrollo del Buen Vivir, Plan Nacional de Ciencia, Tecnología, Innovación y Saberes Ancestrales, Transformación de la Matriz Productiva y los Objetivos de Desarrollo del Milenio, Plan Zonal 3, Plan Estratégico de la Provincia de Chimborazo, Plan de Desarrollo Cantonal, normativa que se deben considerar como referentes fundamentales con una metodología que responda a la demanda de la profesión, al mercado laboral y al campo ocupacional, en concordancia con el Modelo Educativo y Pedagógico de la Universidad Nacional de Chimborazo, cuyos objetivos fundamentales es el de garantizar aprendizajes significativos.

La gestión del conocimiento universitario ha sido una de las mayores preocupaciones de 
los académicos (Rivera, 2000; Espinoza, 1999; Sander, 1996, Pérez Lindo, 1985).

(Perez, 2017) en su obra el uso del conocimiento en la Universidad, hace un aporte significativo al manifestar que la gestión del conocimiento debe apuntalar a crear una nueva sociedad, inteligente y solidaria, es decir, con uso intensivo de las ciencias y las tecnologías, considera una acción intencional y pragmática para resolver problemas aprovechando el uso de la información y del conocimiento científico, estos avances deben ser aprovechados por quienes practicamos la docencia utilizando nuevas estrategias metodológicas que hagan de las clases más activas, reflexivas y críticas tendiente a fortalecer el desarrollo de competencias en los estudiantes.

Jabif señala que "La enseñanza requiere una vinculación explícita entre la actividad del profesor y la del estudiante" (Jabif, 2007). En este sentido, el conocimiento se debate en nuevos escenarios junto a una metodología didáctica, donde el interés hacia el estudio, favorece el proceso de enseñanza-aprendizaje de forma activa y continua y, por lo tanto, se posibilita al cumplimiento de la vinculación en concordancia con las prácticas de los estudiantes y la organización del trabajo de los docentes. (Santoveña, 2013)

El presente trabajo tiene por objeto analizar la Metodología de enseñanza docente en el proceso educativo de la carrera de Pedagogía de la Historia y las Ciencias Sociales, considerando estrategias metodológicas de enseñanza aprendizaje con el uso de la TIC y la aplicación de las TAC que integren la historia y las ciencias sociales, a partir de las vivencias de los actores educativos, orientados a promover proyectos colaborativos, que prioricen la práctica de la investigación-acción, formación, vinculación participativa, en atención a la diversidad, equidad, en el marco del contexto dialógico y cultural.

\section{DESARROLLO CONCEPTUAL}

En principio la metodología docente supone una manera concreta de liderar el aprendizaje, con métodos adecuados y una herramienta concreta que se utiliza para transmitir los contenidos, procedimientos $\mathrm{y}$ principios al estudiante $\mathrm{y}$ que se cumplan los objetivos de aprendizaje propuestos. (Hernández, 2008)

Pero, elegir una forma de enseñar frente a otra no es casual ni aleatoria sino que depende de diversos factores, como los objetivos, contenidos, la experiencia previa del docente, y las concepciones propias sobre lo que supone enseñar o aprender, así por ejemplo, si un docente concibe que aprender supone escuchar conceptos establecidos y que enseñar supone transmitirlos elegirá una metodología más expositiva que otro docente que concibe que el alumnado tienen conocimientos previos $y$ que enseñar supone ayudar a que el alumnado descubra o se interrogue por ellos.

La metodología, en ese sentido, juega un papel fundamental por cuanto una de las diferencias básicas entre unos métodos didácticos y otros es justamente la presencia y el papel de los docentes. (Beraza, 2011) La pedagogía utilizada supone un gran protagonismo del docente, que incluye trabajo, trabajo autónomo del estudiante y trabajo investigativo o de campo. Esa progresión en la metodología debería ser patente en el diseño de los planes de estudio y en el desarrollo de las asignaturas de acuerdo a la malla curricular. 
Tabla 1. Relación de concepciones y metodología

\begin{tabular}{|l|l|}
\hline \multicolumn{1}{|c|}{ Si entendemos que } & \multicolumn{1}{|c|}{ Entonces la metodología consistirá } \\
\hline $\begin{array}{l}\text { El aprendizaje consiste en que el alumnado } \\
\text { adquiera una serie de respuestas o } \\
\text { habilidades... }\end{array}$ & ..en centrarse en los ejercicios y la práctica \\
\hline $\begin{array}{l}\text { El aprendizaje consiste en adquirir } \\
\text { conocimientos }\end{array}$ & $\begin{array}{l}\text {.. en la exposición de contenidos para que el } \\
\text { alumnado los adquiera }\end{array}$ \\
\hline $\begin{array}{l}\text { El aprendizaje consiste en la construcción del } \\
\text { conocimiento por parte del alumnado,.. }\end{array}$ & $\begin{array}{l}\ldots \text { en la interacción y el fomentar la participación } \\
\text { del alumnado, generando reflexiones sobre la } \\
\text { realidad y dándoles posibilidades de experiencia } \\
\text { y contraste con lo que enseñamos }\end{array}$ \\
\hline
\end{tabular}

Fuente: (Hernánez, 1997)

Si es cierto que el Modelo educativo de una universidad encarna su filosofía educativa, la ambición de su emprendimiento educativo y la trascendencia de su misión ante la comunidad, resulta absolutamente fundamental insistir en la dimensión de coherencia paradigmática que debe atravesar la totalidad de este edificio conceptual, y reflejarse así en todas las carreras, en el desempeño de todos los miembros de la institución, directivos, administrativos, educadores, estudiantes (Chimborazo, 2014). A menudo se considera en efecto la cuestión didáctico-curricular como mero asunto técnico, metodológico, que consiste en aplicar con constancia una serie de etapas predefinidas, cuando se trata en realidad de un asunto altamente filosófico, que implica una respuesta ordenada a tres tipos de respuestas:

Las orientaciones metodológicas en que se sustenta la Carrera en Pedagogía de la Historia y las Ciencias Sociales se basa en la vinculación entre teoría y práctica para motivar a los estudiantes en la utilización de métodos fuera de lo convencional como:

- $\quad$ Aprendizaje Basado en Problemas cuyo punto de partida es un problema que, diseñado por el educador, el estudiante en grupos de trabajo ha de abordar de forma ordenada y coordinada las fases que implican la resolución o desarrollo del trabajo en torno al problema o situación.

- $\quad$ Aprendizaje por proyectos: Los estudiantes llevan a cabo la realización de un proyecto en un tiempo determinado para resolver un problema o abordar una tarea mediante la planificación, diseño y realización de una serie de actividades y todo ello a partir del desarrollo y aplicación de aprendizajes adquiridos y del uso efectivo de recursos.

- $\quad$ Aprendizaje cooperativo: Enfoque interactivo de organización del trabajo en el aula en el cual los educandos son responsables de su aprendizaje y del de sus compañeros en una estrategia de corresponsabilidad para alcanzar metas e incentivos grupales.

- El aprendizaje por descubrimiento inductivo. - El aprendizaje se convertirá como ente generador de descubrimiento inductivo y autónomo, colocando el acento en el valor motivacional de la experiencia directa, en el descubrir por sí mismo, con énfasis en la observación y en la experimentación metodología utilizada para el estudio de la geografía.

Se plantea una Metodología de situaciónproblema en el aprendizaje de la Historia:

- $\quad$ Método Narrativo Interrogativo: método direccionado para el estudio de las ciencias 
sociales permite narrar, "vive experimenta ", en forma clara y precisa, estableciendo lo positivo y negativo de los hechos y fenómenos percibidos en la narración.

- $\quad$ Método Estudio de Casos: le permite al estudiante que construya el conocimiento en base a un problema histórico, o social, permitiendo elaborar una explicación histórica del tema de estudio.

- Método Científico: permite que el estudiante razone y prediga, ideas sobre la experimentación planificada y los modos de comunicar los resultados experimentales y teóricos.

Además, se considerará la epistemología de la investigación en todos los semestres de acuerdo a su complejidad; Investigación acción participativa: Lesson study, historias de vida, observación y exploración.

El objetivo de la investigación es utilizar un enfoque de gestión de conocimiento basado en las bondades del Moodle para contribuir en el proceso de aprendizaje con el acceso a una plataforma gratuita que permita la interacción docente - estudiantes- sociedad virtual.

\section{DESARROLLO METODOLÓGICO}

La carrera de en Pedagogía de la Historia $y$ las Ciencias Sociales se fundamenta en el ámbito disciplinar, pedagógico-didáctico, donde se desarrolla competencias orientadas a una realidad histórica, social, económico y geopolítico y espacial, así como la integración en la aplicación de las TIC y las TAC, la investigación-acción, formación y vinculación participativa.

Los docentes se convierten en actores claves en la construcción del buen vivir, promoviendo la integración recíproca entre la educación, el sector productivo y la investigación científica y tecnológica; fortaleciendo la cultura investigativa, práctica; de los estudiantes a través de horas de investigación a partir de un proceso secuencial de primero a noveno, deben generar espacios que fortalezcan la academia, en la investigación y documentación de la memoria social, el patrimonio cultural y los conocimientos diversos, para estimar de esta manera la riqueza cultural, la diversidad y la pluriculturalidad, partiendo de la diversidad que se presenta en el aula y en la comunidad de estudiantes, posibilitando de esta manera el cumplimiento fomentando el diálogo de saberes entre el contexto y la educación.

Se ha de considerar que el maestro es la persona que debe integrar la tecnología en procesos de empoderamiento de las instituciones educativas, con el fin de lograr su gestión, mantenimiento y mejoramiento permanente, lo que permita cada vez ampliar la cobertura educativa.

Los horizontes epistemológicos forman parte de las actuales tendencias del siglo XXI; ante esta complejidad se crea la necesidad de reorientar un conocimiento teórico-práctico y metodológico en la Carrera en Pedagogía de la Historia y las Ciencias Sociales, que sustenten el rediseño curricular, con los paradigmas que prevalecen en la historia y las ciencias sociales y la integración de disciplinas, cambios tecnológicos en respuesta al contexto socio económico y la formación del ser humano íntegro.

La formación profesional, superará el paradigma tradicional y para lograr su fin se debe enfocar en una metodología participativa, en la construcción de la cohesión social, la democracia, por otro lado, el horizonte holístico es considerado como otro pilar fundamental en la carrera, se debe reconocer con cierta facilidad las relaciones que existen entre las diferentes asignaturas y el mundo que les rodea, la adaptabilidad a situaciones nuevas, combinar los conocimientos pertinentes con la inteligencia práctica social, a la hora de resolver problemas reales por sí mismo. 
Partiendo de lo analizado, en la Carrera de Pedagogía de la Historia y las Ciencias Sociales existen docentes con muchos años de experiencia, sin embargo tras ser una ventaja de destacar también puede ser que se formaron con modelos pedagógicos diferentes a las necesidades actuales de nuestro contexto, ello implica que no puedan desarrollar en sus estudiantes, las competencias necesarias para contribuir con el perfil de egreso, puesto que continúan con una metodología caduca, y esto conlleva a observar que no existe consistencia metodológica entre objetivos, contenidos, actividades y evaluación.

De una encuesta realizada a los estudiantes de la carrera se evidencia que los docentes de la carrera plantean una metodología caracterizada por mayor protagonismo del docente y menor del alumnado, en un $40 \%$, mientras que en el $60 \%$ se sitúan una metodología donde el protagonismo del profesor es mayor que del estudiante.

\section{IMPLEMENTACIÓN PLATAFORMA MOODLE}

El aula virtual de la asignatura de Geografía Física, creada:

Figuras N.1, 2, 3: Contenido aula virtual Geografía Física

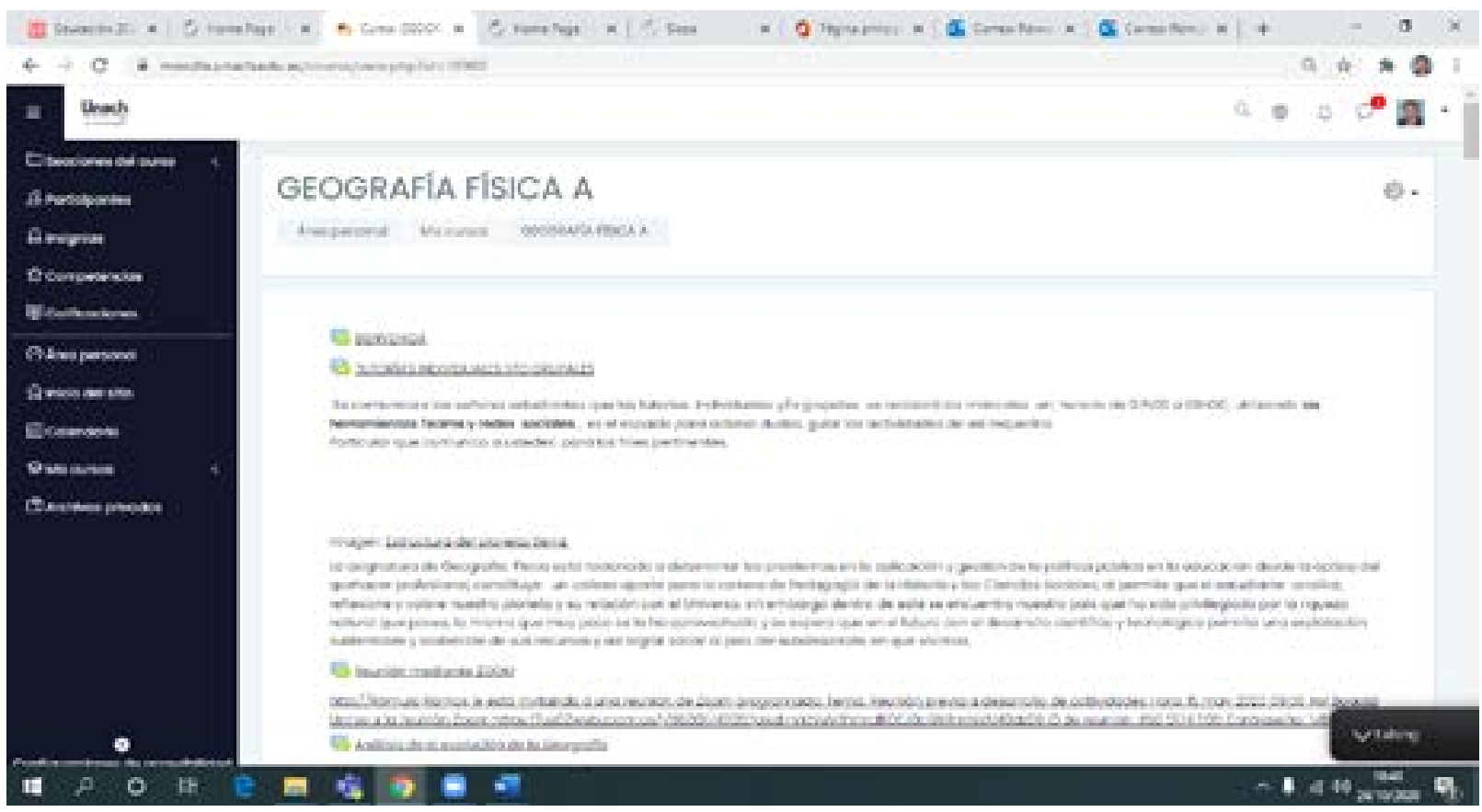



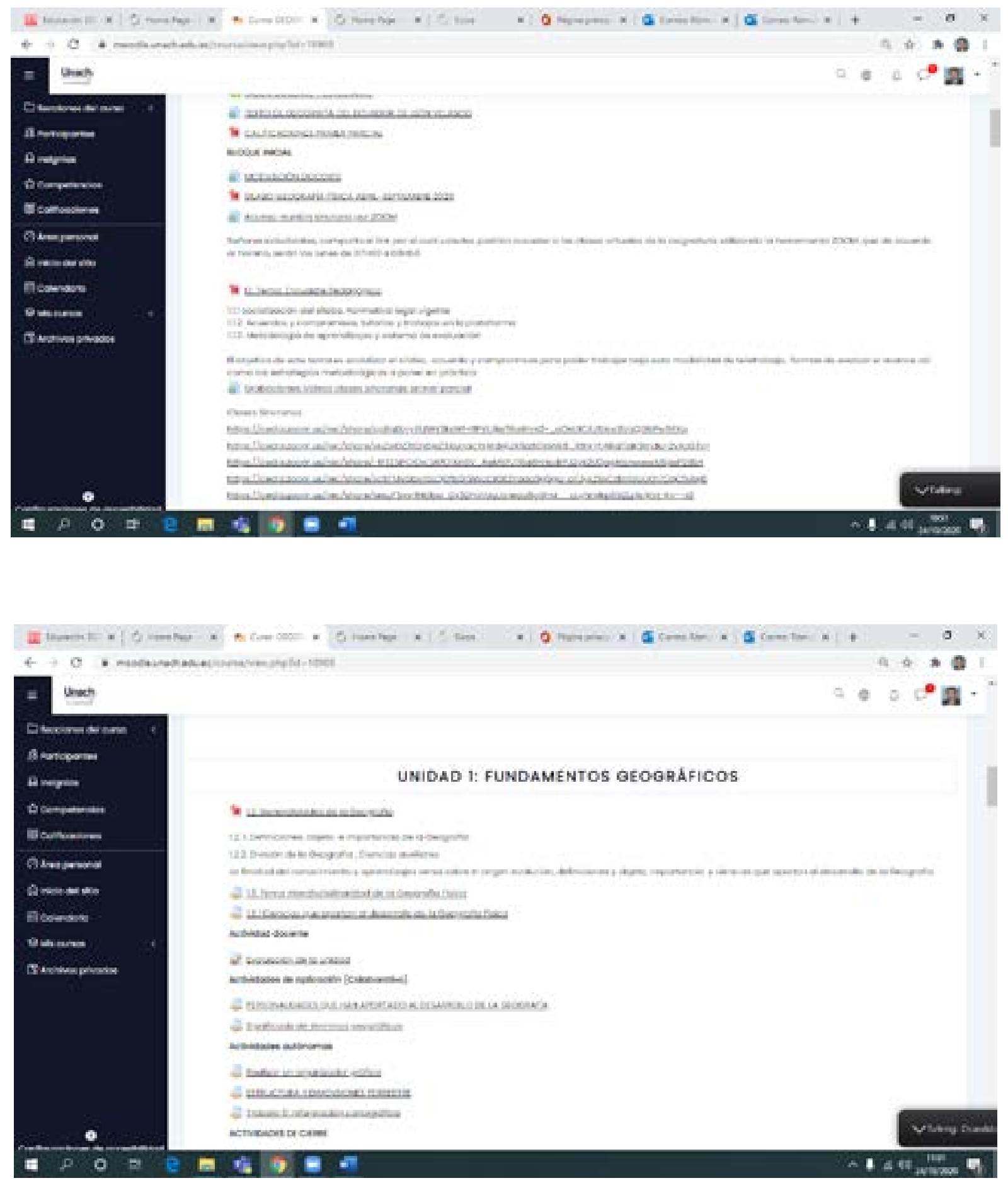

Fuente: https://moodle.unach.edu.ec/ 
El campus virtual de la Universidad Nacional de Chimborazo mediante la dirección electrónica: https://moodle.unach.edu.ec/, se desarrolla bajo la plataforma Moodle, que es una herramienta que ayuda a la gestión de Aprendizaje. Esta plataforma virtual está generalmente compuesta por módulos con funciones independientes que incluye todo lo necesario para poder ofrecer cursos a través de internet o una intranet. La integración es su principal valor, dado que todas las utilidades mencionadas ya existen por separado fuera de las plataformas. A nivel pedagógico Moodle, ofrece funcionalidades bastante atractivas para los docentes, promueve el constructivismo, fomenta el trabajo colaborativo, su interfaz es sencilla y amigable, y pretende ser una alternativa o complemento para las clases presenciales que ejecuta el docente, denominándose a esta mezcla de modalidades, tanto presencial y virtual como Blended Learning (b-learning), que es una estrategia educativa en la que se integran actividades y recursos de estas dos modalidades en diferentes proporciones, para lograr los objetivos de un curso o asignatura, con mayor eficiencia y calidad. (Solís, Silva, \& Humanante, 2018)

Gestionar el aula virtual de una asignatura, implica definir aspectos generales a diseñar y la organización necesaria en la creación del curso en línea, que contempla: definir los temas que se van a abordar (contenidos), definir el perfil de los participantes, el cronograma del curso, y la distribución de las actividades a diseñar. Sin embargo en cursos sencillos es el profesor quien hace todas las tareas, no así en cursos de mayor entidad, donde interviene un equipo multidisciplinar es decir la participación de pedagogos, psicólogos, técnicos y diseñadores gráficos (Silva Quiroz, 2011)"publisher":"Editorial UOC","numberof-pages": "167", "source": "Google Books","abstract":"El libro es una invitación a pensar y reflexionar cómo crear nuevos escenarios formativos mediados por las tecnologías de la información y comunicación, que permitan innovar en la enseñanzaaprendizaje acercándola a las necesidades de la sociedad de la información y las expectativas educativas de las nuevas generaciones. Estudiantes inmersos en una cultura digital, en la cual la interacción, la colaboración y la construcción conjunta, es parte natural de sus prácticas y formas de acceder a la información y aprender. El libro recoge aspectos teóricos relacionados a la formación virtual, los EVA y la tutoría online, presenta modelos concretos y probados para diseñar y moderarlos. Es una guía para todos aquellos profesionales vinculados a la educación interesados en transformar la docencia insertando la virtualidad como una forma de apoyar, complementar o reemplazar la formación presencial.","ISBN":"978-84-9788963-6","note":"Google-Books-ID: _OdFFeq_wb MC","language":"es","author":[\{“family":"Silva Qu iroz","given":"Juan"\}],"issued":\{“date-parts":[["2 011"]]\}\}\}],"schema":"https://github.com/citationstyle-language/schema/raw/master/csl-citation. json"\} .

Uno de los primeros diseños instruccionales aplicados por los docentes a nivel de Latinoamérica para la gestión de un aula virtual está la metodología PACIE. Esta metodología reúne elementos como la motivación y el acompañamiento que enriquecen la educación en línea, dando realce a la calidad en los procesos sociales comunicacionales para brindar apoyo al compartir académico y al aprendizaje sociocultural perteneciente al entorno virtual, que se fundamenta en un constante levantar la autoestima de quienes toman la decisión de incluir la educación tanto a distancia como semipresencial (Blearning) como una alternativa para su formación. (Perdómo \& Beltrán, 2014). En este espacio es donde se integran herramientas que facilitan los procesos educativos presenciales en concordancia con el aprendizaje en línea como es el caso 
específico de la asignatura de Geografía Física correspondiente al tercer semestre de la Carrera de Pedagogía de la Historia y las Ciencias Sociales.

Bajo este análisis se ha considerado incluir dentro de la gestión del aula virtual recursos o actividades tales como el chat, foros, contenidos hipertextuales, vídeos, enlaces a bibliografía o sitios referenciales, cuestionarios mediante formularios embebidos o programados en el mismo sitio como parte de la evaluación sumativa. Toda esta información subida y generada tanto por el docente como por los estudiantes, al finalizar el período académico permitirá la elaboración de reactivos y creación del portafolio.

\section{CARACTERÍSTICAS PRINCIPALES:}

- $\quad$ Plataforma institucional

- Utiliza internet

- $\quad$ Acceso desde cualquier punto de red

- Es gratis y no necesita software de instalación

- Los recursos creados permanecerán con acceso a los estudiantes las 24 horas

- $\quad$ Permite la descarga de los recursos

- $\quad$ Genera recursos de manera inmediata

- Los recursos generados se pueden imprimir y reproducir en cualquier navegador o de manera local desde diferentes medios de almacenamiento

\section{CONCLUSIÓN:}

El aula virtual creada con una nueva metodología de trabajo docente que permite el uso de las TIC como un soporte a los procesos de aprendizaje y autoaprendizaje, dará realce al esquema pedagógico de la educación que se da actualmente, puesto que toma como elementos esenciales recursos multimedia que motiva el aprendizaje.

Sin duda hay obstáculos que van surgiendo como que los estudiantes no están adiestrados en el cumplimiento del trabajo colaborativo, o no quieren participan en la utilización de los recursos disponibles. Pero estamos seguros que la estrategia planteada crea nuevas expectativas tanto para el docente como para el estudiante.

\section{REFERENCIAS BIBLIOGRÁFICAS}

Beraza, M. Á. (2011). Metodología docente.Teaching Methodology. Revista de Docencia Universitaria, Vol.9 (3), 75-98.

Calero, M. (2008). Constructivismo Pedagógico: Teorías y aplicaciones Básicas. Madrid: Alfaomega, pag. 174, ISBN: 9789701513866.

Carvajal, B. C. (2011). Gestión del conocimiento sustentable universitario, Visión aproximada de experiencias latinoamericanas . Bogotá: HALLAZGOS / ISSN: 1794-3841 / , N. ${ }^{\circ} 22$ / Universidad Santo Tomás / pp. 159-181.

Chimborazo, U. N. (2014). Recuperado el 10 de 05 de 2017, de http://www.unach.edu.ec/ images/pdf/descargas/modelo_educativo_y_ pedagogico_de_la_unach_2014_aprobado_2_ instancia_hcu.pdf

Hernández, J. (2008). Metodología de enseñanza y aprendizaje en altas capaciddes. México : Trillas.

Hernánez, P. (1997). Una sistematización de procedimientos para la educación afecto adaptiva en clase. Madrid: Narcea.

Jabif, L. (2007). "La docencia universitaria bajo enfoque por competencias" Chile: $\mathrm{UACH}$; Imprenta Austral. Chile: UACH; Imprenta Austral.

Ministerio de Educación y Cultura. (2010). Hacia el Plan Decenal de Educación del Ecuador 20062015. Quito: Consejo Nacional de Educación. 
Perez, L. (2017). El uso del conocimiento en la Universidad. Buenos Aires: Teseo.

Rodríguez A.,Araujo A., Urrutia Javier. (2001). La gesti-n del conocimiento científico- técnico en la universidad: un caso y un proyecto. Universidad del País Vasco (UPV/EHU): Cuadernos de Gesti-n Vol. 1. N.1/4 1.

Universidad Ncional de Chimborazo. (2014). Recuperado el 10 de 05 de 2017, de http://www. unach.edu.ec/images/pdf/descargas/modelo_ educativo_y_pedagogico_de_la_unach_2014_ aprobado_2_instancia_hcu.pdf 